\title{
Equidimensionality of the Brauer loop scheme
}

\author{
Brian Rothbach \\ University of California, Berkeley \\ rothbach@math. berkeley.edu
}

Submitted: Aug 5, 2009; Accepted: May 11, 2010; Published: May 20, 2010

Mathematics Subject Classification: 14M99

\begin{abstract}
We give another description of certain subvarieties of the Brauer loop scheme of Knutson and Zinn-Justin. As a consequence, we show that the Brauer loop scheme is equidimensional.
\end{abstract}

\section{Contents}

1 Introduction $\quad 1$

2 A decomposition and the dimension of the $F_{\pi}$ 's $\quad 3$

3 A geometric description $\quad 4$

4 A cyclic action on the $\overline{F_{\pi}} \quad 8$

5 Equations for the $\overline{F_{\pi}}$ 's $\quad 9$

\section{Introduction}

Let $N$ be a positive integer. An integer sequence $\left(i_{1}, \ldots, i_{k}\right) \in\{1, \ldots, N\}^{k}$ is said to be cyclically ordered if either $i_{1}=i_{2}=\cdots=i_{k}$, or $i_{1} \neq i_{k}$ and for some $1 \leqslant l \leqslant k$, the cyclically rotated sequence $\left(i_{l}, i_{l+1}, \ldots, i_{k}, i_{1}, \ldots, i_{l-1}\right)$ is weakly increasing. We will write $\circlearrowleft\left(i_{1}, \ldots, i_{k}\right)$ as shorthand for the statement "the sequence $\left(i_{1}, \ldots, i_{k}\right)$ is cyclically ordered".

Knutson and Zinn-Justin [1] defined a nonstandard multiplication $\bullet$ on $M_{N}(\mathbb{C})$, the set of $N \times N$ complex matrices, by setting $(P \bullet Q)_{i k}=\sum_{j: \circlearrowleft(i, j, k)} P_{i j} Q_{j k}$. We refer to their paper as a reference for several nice geometric models of this multiplication. We recall the following facts from their paper. 
1. A matrix $M$ is invertible under $\bullet$ if and only if the diagonal entries are nonzero. The set of invertible matrices under $\bullet$ is a solvable Lie group, with the invertible diagonal matrices $T$ serving as a maximal torus, and with unipotent radical $U$ the set of all matrices with ones along the diagonal.

2. Let $E=\left\{M \bullet M=0: M \in M_{N}(\mathbb{C})\right\}$, which can be described set theoretically by the (possibly nonreduced) equations $(M \bullet M)_{i j}=0$ for $1 \leqslant i, j \leqslant N$ and $M_{i i}=0$ for $1 \leqslant i \leqslant N$. Then

$$
E=\coprod_{\pi \in \mathfrak{I}} F_{\pi}
$$

where $\mathfrak{I} \subset S_{N}$ is the set of involutions in $S_{n}$, and for each $\pi \in \mathfrak{I}, F_{\pi}$ is the set of all matrices $M \in E$ such that the upper triangular part of $M$ is Borel conjugate to the strictly upper triangular part of $\pi$.

3. Each $F_{\pi}$ is a union of $(U, \bullet)$ orbits; in other words, $U \bullet F_{\pi}=F_{\pi}$.

4. Suppose $\pi$ has $k$ fixed points. Then $F_{\pi}$ is nonempty and irreducible of dimension $\frac{1}{2}\left(N^{2}-k\right)$.

As a consequence, Knutson and Zinn-Justin were able to classify all the top dimensional irreducible components of $E$ and to give a partial set of equations for the top dimensional components of $E$. Moreover, they compute the multidegree of these top dimensional components and connect that polynomial to the entries of the Frobenius-Perron eigenvector of a certain Markov process associated to the Brauer loop model.

The main theorem of this paper is a proof of the following conjecture of Knutson and Zinn-Justin.

Conjecture 1 The Brauer loop scheme is equidimensional; that is the irreducible components of $E$ are exactly $E_{\pi}=\overline{F_{\pi}}$ where $\pi \in S_{N}$ is an involution with maximal number of 2-cycles. In particular, $E$ is equidimensional of dimension $\left\lfloor N^{2} / 2\right\rfloor$.

Our method for proving this conjecture is to generalize a construction of Knutson and Zinn-Justin that gives a dense subvariety $G_{\pi}$ of $F_{\pi}$ for any involution $\pi$. As a consequence, we can generalize the equations for the top dimensional components and also prove the following characterization of the closure poset of the $F_{\pi}$ 's.

Theorem. Let $\pi, \pi^{\prime}$ be two involutions in $S_{N}$, and suppose that $\pi$ has $k$ 2-cycles $\left(i_{1}, j_{1}\right), \ldots\left(i_{k}, j_{k}\right)$ and $N-2 k$ fixed points $1 \leqslant a_{1}<a_{2}<\cdots<a_{N-2 k} \leqslant N$. Then $F_{\pi} \subset \overline{F_{\pi^{\prime}}}$ if and only if of $\pi^{\prime}$.

a. Every two cycle $\left(i_{l}, j_{l}\right)(1 \leqslant l \leqslant k)$ of $\pi$ occurs in the disjoint cycle decomposition

b. Every two cycle occurring in the disjoint cycle decomposition of $\pi^{\prime}$ is either of the form $\left(i_{l}, j_{l}\right)$ or of the form $\left(a_{i}, a_{N-2 k+1-i}\right)$ for some $1 \leqslant i \leqslant\left\lfloor\frac{N-2 k}{2}\right\rfloor$.

The paper proceeds as follows. Section 2 reviews the decomposition of the Brauer loop scheme into the finitely many irreducible locally closed schemes $F_{\pi}$. In section 3 , 
we generalize a theorem of Knutson and Zinn-Justin to obtain for each involution $\pi$ a parameterization of a dense subvariety $G_{\pi}$ of $\overline{F_{\pi}}$. In section 4 , we take a quick digression to analyze the effects of a natural cyclic action. In section 5, we show how to construct a partial set of equations for each $F_{\pi}$, and use this to characterize the closure poset of the $F_{\pi}$ 's. The conjecture of Knutson and Zinn-Justin is an immediately corollary of the classification of the poset.

\section{A decomposition and the dimension of the $F_{\pi}$ 's}

Given a matrix $M$, we define $M_{\leqslant}$to be the upper triangular matrix associated to $M$; namely $\left(M_{\leqslant}\right)_{i j}=M_{i j}$ if $i \leqslant j$, and $\left(M_{\leqslant}\right)_{i j}=0$ otherwise. Similarly, we will write $M_{<}$ and $M_{>}$to refer to the strictly upper triangular matrix associated to $M$ and the strictly lower triangular matrix associated to $M$ respectively. Notice that $M=M_{\leqslant}+M_{>}$for any matrix $M$.

Recall that $E=\{M: M \bullet M=0\}$. From the definition of $\bullet, M \in E$ if and only if $M_{\leqslant}^{2}=0$ and $M_{\leqslant} M_{>}+M_{>} M_{\leqslant}$is upper triangular. (The alternative characterizations of - given in [1] make this more apparent.) In particular, one can characterize the matrices $M_{\leqslant}$arising from $M \in E$ by the following theorem of Melnikov [2].

Theorem 1 Let $B \subset G L_{N}$ be the Borel subgroup of invertible upper triangular matrices. Then $B$ acts by conjugation (under ordinary matrix multiplication) on the set $V=\{L: L$ is upper triangular and $\left.L^{2}=0\right\}$. Under this action, $V$ decomposes into a finite union of $B$ orbits, indexed bijectively by involutions $\pi \in S_{N}$. The $B$ orbit associated to $\pi$ is $B \cdot \pi_{<}$.

For each involution $\pi \in S_{N}$, define the locally closed subset $F_{\pi}$ of $E$ to be $\{M$ : $M \bullet M=0$ and $\left.M_{\leqslant} \in B \cdot \pi_{<}\right\}$. We have the following results from Knutson and ZinnJustin.

Theorem 2 Let $\mathfrak{I} \subset S_{N}$ be the set of all involutions. Then,

1. $E=\coprod_{\pi \in \mathfrak{I}} F_{\pi}$

2. Each $F_{\pi}$ is a union of $(U, \bullet)$ orbits.

3. Suppose $\pi$ has $k$ fixed points. Then $F_{\pi}$ is nonempty and irreducible of dimension $\frac{1}{2}\left(N^{2}-k\right)$.

Since $E=\bigcup_{\pi \in \mathfrak{I}} \overline{F_{\pi}}$ decomposes into a union of finitely many irreducible closed subvarieties, we can immediately make the following observation about $E$.

Corollary 1 The only possible irreducible components of $E$ are the varieties $\overline{F_{\pi}}$ and the top dimensional components correspond bijectively with involutions having a maximal number of two cycles.

Conceivably the lower dimensional $\overline{F_{\pi}}$ 's could also be irreducible components of $E$. The point of the rest of the paper is to show that each of these subvarieties is contained in some top dimensional component. 


\section{A geometric description}

Our next goal is to give a geometric description of the varieties $\overline{F_{\pi}}$; we will see that each such variety is the closure of the $\bullet$ conjugation orbit of a torus invariant subspace. This construction generalizes the parameterization of top dimensional components developed by Knutson and Zinn-Justin.

Let $\pi \in S_{N}$ be an involution with $k$ 2-cycles $\left(i_{1}, j_{1}\right), \ldots,\left(i_{k}, j_{k}\right)$, where $i_{l}<j_{l}$ for all $1 \leqslant l \leqslant k$, and $N-2 k$ fixed points $a_{1}<a_{2}<\cdots<a_{N-2 k}$. We define a matrix $\underline{\pi}$ as follows.

1. If $i$ is not a fixed point of $\pi, \underline{\pi}_{i, m}=\delta_{\pi(i), m}$.

2. For $\left\lfloor\frac{N-2 k+1}{2}\right\rfloor+1 \leqslant l \leqslant N-2 k, \underline{\pi}_{a_{l}, m}=\delta_{a_{N-2 k+1-l}, m}$.

3. For $1 \leqslant l \leqslant\left\lfloor\frac{N-2 k+1}{2}\right\rfloor, \underline{\pi}_{a_{l}, m}=0$.

\section{Examples.}

1. If $N$ is even and $\pi$ has a maximal number of two cycles, then $\underline{\pi}$ is the permutation matrix of $\pi$. If $N$ is odd and $\pi$ has a maximal number of two cycles, then $\underline{\pi}$ is the permutation matrix of $\pi$ with the unique nonzero diagonal entry replaced by zero.

2 . If $\pi=i d_{N}$, then $\underline{\pi}$ is just $\left(w_{0}\right)_{>}$, where $w_{0}$ is the matrix with 1 's on the antidiagonal and zeroes elsewhere. For example, $i d_{4}$ is given by

$$
\left(\begin{array}{llll}
0 & 0 & 0 & 0 \\
0 & 0 & 0 & 0 \\
0 & 1 & 0 & 0 \\
1 & 0 & 0 & 0
\end{array}\right)
$$

3. In general, one can obtain $\underline{\pi}$ from $\pi$ by replacing the $k \times k$ square submatrix whose rows and columns are the fixed points of $\pi$ with $\left(w_{0}\right)_{>}$, where $w_{0}$ again only has 1 's on the antidiagonal. For example, if $\pi=(12) \in S_{4}$, then $(12)$ is given by

$$
\left(\begin{array}{llll}
0 & 1 & 0 & 0 \\
1 & 0 & 0 & 0 \\
0 & 0 & 0 & 0 \\
0 & 0 & 1 & 0
\end{array}\right)
$$

Recall that the group of $\bullet$ invertible matrices contains a torus $T$ given by the set of all invertible diagonal matrices and a unipotent factor $U$ given by the set of all matrices with ones on the diagonals. For any element $t \in T$, we will write $t_{i}$ as shorthand for $t_{i i}$, $1 \leqslant i \leqslant N$.

We are now ready to construct for each involution $\pi \in S_{N}$ an dense subvariety $G_{\pi}$ of the $\overline{F_{\pi}}$.

Theorem 3 Let $G_{\pi}=U \bullet\{\underline{\pi} t: t \in T\}$ (so the $U$ action is by-conjugation, while $\underline{\pi} t$ is defined in terms of ordinary matrix multiplication.) Then $\overline{F_{\pi}}=\overline{G_{\pi}}$. 
Proof. Direct calculation shows $\underline{\pi} t \in E$ for all $t \in T$. The upper triangular part of $\underline{\pi} t$ is of the form $\pi_{<} t$ by construction, and thus $\underline{\pi} t \subset F_{\pi}$. Since $F_{\pi}$ is invariant under $\bullet$ conjugation by $U, G_{\pi} \subset F_{\pi}$, and thus $\overline{G_{\pi}} \subset \overline{F_{\pi}}$. By the irreducibility of $F_{\pi}$, in order to prove $\overline{G_{\pi}}=\overline{F_{\pi}}$ we merely need to prove that both $F_{\pi}$ and $G_{\pi}$ both have the same dimension $\frac{1}{2}\left(N^{2}-k\right)$.

Recall that the dimension of $F_{\pi}$ was shown to be $\frac{1}{2}\left(N^{2}-k\right)$ for any involution $\pi$ by Knutson and Zinn-Justin [1].

To compute the dimension of $G_{\pi}$, we generalize an argument of Knutson and ZinnJustin. First, we compute the dimension of the $(U, \bullet)$-orbit of a generic point in $\{\underline{\pi} t$ : $t \in T\}$. Then we show the elements of $\{\underline{\pi} t: t \in T\}$ correspond to distinct $U$ orbits, so that the dimension of $G_{\pi}$ is the dimension of the generic orbit plus the dimension of $\{\underline{\pi} t: t \in T\}$.

We compute the dimension of the generic orbit by finding the size of the $U$-stabilizer. Let $\mathfrak{U}=\left\{M \in M_{N}(\mathbb{C}): M_{i i}=0\right\}$ denote the Lie algebra of $U$. In order to compute the dimension of the $U$ stabilizer of $\underline{\pi} t$, it suffices to find the stabilizer of $\underline{\pi} t$ in $\mathfrak{U}$. Equivalently, we must find the dimension of the solution space of $\underline{\pi t} \bullet P=P \bullet \underline{\pi t}$ where $P \in \mathfrak{U}$. Note that then the dimension of the generic orbit will be equal to the generic number of linearly independent equations arising from the condition $\underline{\pi} t \bullet P=P \bullet \underline{\pi t}$.

Associate to $\underline{\pi}$ a partially directed link diagram $L_{\underline{\pi}}$ as follows: If $i$ is not a fixed point of $\pi$, then connect the points $i$ and $\pi(i)$ with an undirected edge. Recall that we have labeled the fixed points of $\pi$ as $a_{1}<a_{2}<\cdots<a_{N-2 k}$; to complete the partially directed link diagram, for each $\left\lfloor\frac{N-2 k+1}{2}\right\rfloor+1 \leqslant l \leqslant N-2 k$ draw a directed edge from $a_{l}$ to $a_{N-2 k+1-l}$ for all $\left\lfloor\frac{N-2 k}{2}\right\rfloor \leqslant l \leqslant N-2 k$ (the arrow should point from the larger value to the smaller value). Note that if $N$ is odd, there will be a unique fixed point in the diagram.

We make a few observations about the resulting diagrams. If $i_{1}<i_{2}<i_{3}<i_{4}$ contain a pair of directed arrows, then those arrows do not cross and consist of an arrow pointing from $i_{4}$ to $i_{1}$ and an arrow pointing from $i_{3}$ to $i_{2}$. Similarly, if $i_{1}<i_{2}<i_{3}$ consist of a directed arrow and the unique fixed point, then the directed arrow points from $i_{3}$ to $i_{1}$, and $i_{2}$ is the fixed point.

In order to simplify notation in the upcoming discussion, we will introduce the involution $\pi^{\prime}$ associated to the link diagram obtained by replacing all directed edges in $L_{\underline{\pi}}$ with undirected edges; the main convenience is that an edge connects two distinct points $i, j$ in the link diagram $L_{\pi}$ if and only if $\pi^{\prime}(i)=j$. In addition, $\underline{\pi} t$ can be obtained from $\pi^{\prime} t$ by setting $t_{i}=0$ for each $i$ that is the tail of a directed edge and setting $t_{i}=0$ for the unique fixed point of $\pi^{\prime}$ if $N$ is odd. Similarly the equations for the stabilizer of $\underline{\pi} t$ can be obtained from those of $\pi^{\prime} t$ by setting the same $t_{i}$ 's equal to 0 .

The equation arising from $\pi^{\prime} t \bullet P=P \bullet \pi^{\prime} t$ in coordinate $(i, j)$ is of the form

$$
t_{\pi^{\prime}(i)} P_{\pi^{\prime}(i) j}\left[\circlearrowleft\left(i \leqslant \pi^{\prime}(i) \leqslant j\right)\right]=P_{i \pi^{\prime}(j)} t_{j}\left[\circlearrowleft\left(i \leqslant \pi^{\prime}(j) \leqslant j\right)\right]
$$

where $1 \leqslant i, j \leqslant N$ and $[S]$ is defined by $[S]=1$ if $S$ is a true statement and $[S]=0$ otherwise. As previously observed, the equations for the stabilizer of $\underline{\pi} t$ are obtained from 
the equations for the stabilizer of $\pi^{\prime} t$ by setting $t_{i}=0$ for all $i$ at the tail of a directed edge and for the unique fixed point of the link diagram when $N$ is odd.

After setting the appropriate $t$ 's to 0 , the equations corresponding to $i=j$ or $i=\pi^{\prime}(j)$ hold trivially, since either the logical condition is 0 or we have set $t_{i}=t_{j}=0$. So we can assume that $i$ and $j$ lie on distinct orbits of $\pi^{\prime}$ and we group the equations by the corresponding pair of orbits $\left(i, \pi^{\prime}(i)\right),\left(j, \pi^{\prime}(j)\right)$ (note that if $N$ is odd, the one of these orbits may be a fixed point, but not both.) We will show that each pair of edges of the link diagram contributes four linearly independent equations to the stabilizer of a generic $\underline{\pi} t$, and if $N$ is odd, each pair of an edge and the unique unmatched point generically contributes two linearly independent equations.

Let us start with two crossing edges, so we may assume $\circlearrowleft\left(i<j<\pi^{\prime}(i)<\pi^{\prime}(j)\right)$. Looking first at the stabilizer of $\pi^{\prime} t$, we get the four equations:

$$
\begin{aligned}
& t_{i} P_{i j}=P_{\pi^{\prime}(i) \pi^{\prime}(j)} t_{j} \\
& t_{j} P_{j \pi^{\prime}(i)}=P_{\pi^{\prime}(j) i} t_{\pi^{\prime}(i)} \\
& t_{\pi^{\prime}(i)} P_{\pi^{\prime}(i) \pi^{\prime}(j)}=P_{i j} t_{\pi^{\prime}(j)} \\
& t_{\pi^{\prime}(j)} P_{\pi^{\prime}(j) i}=P_{j \pi^{\prime}(i)} t_{i}
\end{aligned}
$$

These equations are linearly independent unless $t_{i} t_{\pi(i)}=t_{j} t_{\pi(j)}$, and so for a generic choice of $t_{i}$ 's we get four linearly independent equations.

Now we consider what happens to these equations when we set $t_{i}=0$ as described above to get the equations of the stabilizer of $\underline{\pi} t$. By the previous observations, at most one of the crossing edges is directed. No matter which $I$ is at the head of a directed edge, at most one of the $t$ 's will be set equal to 0 . If no $t_{i}$ 's are set equal to 0 , then we will still generically have four linearly independent equations. If exactly one $t_{i}$ is set equal to 0 , then we still have that $t_{i} t_{\pi(i)} \neq t_{j} t_{\pi(j)}$ generically (since one side will be zero, and the other generically nonzero), and so there will still generically be four linearly independent equations as desired.

If we have a pair of edges that do not cross, we can assume $\circlearrowleft\left(i<j<\pi^{\prime}(j)<\pi^{\prime}(i)\right)$. Then for the stabilizer of $\pi^{\prime} t$ we get the following six equations:

(a) $t_{i} P_{i \pi^{\prime}(j)}=P_{\pi^{\prime}(i) j} t_{\pi^{\prime}(j)}$

(b) $t_{\pi^{\prime}(j)} P_{\pi^{\prime}(j) i}=P_{j \pi^{\prime}(i)} t_{i}$

(c) $0=P_{i, j} t_{\pi^{\prime}(j)}$

(d) $t_{i} P_{i j}=0$ 
(e) $0=P_{\pi^{\prime}(j) \pi^{\prime}(i)} t_{i}$

(f) $t_{\pi^{\prime} j} P_{\pi^{\prime}(j) \pi^{\prime}(i)}=0$

Again, we obtain equations of $\underline{\pi} t$ by setting some of the $t_{i}$ 's equal to 0 . Clearly, the pair of equations $(c)$ and $(d)$ contribute at most one linearly independent equation, as does the pair $(e)$ and $(f)$. However, as long as at most one of the edges is directed, so at most one $t$ is equal to zero, the equations $(a)-(f)$ generically contribute four linearly independent equations. Suppose that both edges are directed, so two t's have been set equal to zero. By changing the roles of $i, j, \pi^{\prime}(i)$, and $\pi^{\prime}(j)$ and using our previous observations about link diagrams, we may assume that $i<j<\pi^{\prime}(j)<\pi^{\prime}(i)$, that $t_{\pi^{\prime}(i)}=t_{\pi^{\prime}(j)}=0$ and that $t_{i}$ and $t_{j}$ are nonzero. Then the equations simplify to:

$$
\begin{aligned}
& t_{i} P_{i \pi^{\prime}(j)}=0 \\
& 0=P_{j \pi^{\prime}(i)} t_{i} \\
& t_{i} P_{i j}=0 \\
& 0=P_{\pi^{\prime}(j) \pi^{\prime}(i)} t_{i}
\end{aligned}
$$

which again is generically four linearly independent equations.

Finally, if we have an edge and a fixed point, we may assume that $j$ is the fixed point, so $i \neq \pi^{\prime}(i)$. We may assume $i<j<\pi^{\prime}(i)$ and that $t_{i}$ is nonzero by construction of $\underline{\pi}$. Then we get the two equations for the stabilizer of $\underline{\pi} t$ :

$$
\begin{aligned}
& t_{i} P_{i j}=0 \\
& 0=P_{j \pi^{\prime}(i)} t_{i}
\end{aligned}
$$

which are by construction generically linearly independent.

Note that for each pair of edges and for each pair of and edge an a fixed point, we have found a collection of linear independent equations in the corresponding variables. Since this partitions the variables into distinct nonoverlapping sets, the corresponding sets of equations are all mutually independent.

Let $N=2 n+r$ ( $n$ an integer, $r=0$ or 1$)$. Counting the set of independent equations shows that the dimension of the generic orbit is $4 \frac{n(n-1)}{2}+2 n r=2 n^{2}-2 n+2 n r$. Now, $\frac{1}{2}\left(N^{2}-k\right)=\frac{1}{2}\left(4 n^{2}-4 n r+r^{2}-k\right)=\left(2 n^{2}-2 n+2 n r\right)+(2 n+r-k)=$ the dimension of the generic $U$-orbit of $\underline{\pi} t$ plus the dimension of $\underline{\pi} t$. Thus if we can show that each orbit contains at most one element of $\underline{\pi} t$, we are done.

So suppose $P \bullet \underline{\pi} t=\underline{\pi} t^{\prime} \bullet P$ for some $P \in U$. We must show that $t_{i}=t_{i}^{\prime}$ for all $i$ lying on either an undirected edge or the head of an edge of the corresponding link diagram. 
In either case the equation in entry $\left(\pi^{\prime}(i), i\right)$ reads $P_{\pi^{\prime}(i) i} t_{i}=t_{i} P_{i i}$ and since $P \in U$, one gets $t_{i}=t_{i}^{\prime}$ for the required indices. In particular, $\underline{\pi} t=\underline{\pi} t^{\prime}$ as desired. $\square$

\section{A cyclic action on the $\overline{F_{\pi}}$}

Given an integer $k$, we define $[[k]]$ to be the unique number in $\{1, \ldots, N\}$ such that $k=[[k]] \bmod N$.

Knutson and Zinn-Justin [1] observe that there is a natural continuous cyclic action acting on $M_{N}(\mathbb{C})$ that preserves the nonstandard multiplication $\bullet$, given by sending the matrix $M$ to $c(M)$, where $c(M)_{i j}=M_{[[i-1]],[[j-1]]}$. Such a cyclic rotation preserves the relation $\circlearrowleft$, and thus preserves the multiplication $\bullet$. Alternatively one can visualize this action as a translation in their infinite strip model, which again makes it clear that $c$ is a ring homomorphism. The action $c$ fixes the zero matrix, hence also the variety $E=\{M \mid M \bullet M=0\}$.

While the $\overline{F_{\pi}}$ are not invariant under the action of $c$, Knutson and Zinn-Justin were able to show that $c$ maps top dimensional components of $E$ to other top dimensional components. Moreover, for these top dimensional $\overline{F_{\pi}}, c$ corresponds to rotating the link diagram associated to a $\pi$. $\overline{F_{\pi}}$.

Our goal is to prove the following weaker version of the above statement for general

Theorem 4 Suppose we fix an involution $\pi$ and an integer $d$. Then $c^{d}\left(\overline{F_{\pi}}\right)$ is of the form $\overline{U \bullet\left\{c^{d}(\underline{\pi}) t \mid t \in T\right\}}$. Let $\pi^{*}$ be the unique involution such that $c^{d}(\underline{\pi})_{<}=\pi_{<}^{*}$. Then $c^{d}(\underline{\pi})$ can be obtained from $\pi^{*}$ by setting certain nonzero entries of $\pi^{*}$ to zero. In particular, $c^{d}\left(\overline{F_{\pi}}\right) \subset \overline{F_{\pi^{*}}}$, and $c^{d}(\underline{\pi})=\underline{\pi^{*}}$ if $\operatorname{rank}\left(c^{d}(\underline{\pi})_{<}\right)=\operatorname{rank}\left(\pi_{<}^{*}\right)$.

Proof. Note $c(U)=U$, since $U$ is the set of $\bullet$-invertible matrices, and $c$ fixes the identity. Then $U \bullet\left\{c^{d}(\underline{\pi}) t \mid t \in T\right\}=c^{d}\left(G_{\pi}\right)$ is contained in $c^{d}\left(\overline{F_{\pi}}\right)$, and since $c$ is continuous, taking closures gives us the first statement.

Fix $\pi$, let $L_{\underline{\pi}}$ be the link diagram associated to $\underline{\pi}$, and let $a_{1}<a_{2}<\cdots<a_{N-2 k}$ be the fixed points of $\pi$. We can naturally associate to $c^{d}(\underline{\pi})$ the link diagram $L_{c^{d}(\underline{\pi})}$ obtained by rotating the link diagram $L_{\underline{\pi}} d$ times; we observe that $c^{d}(\underline{\pi})$ is a partial permutation matrix such that $c^{d}(\underline{\pi})_{i j}=1$ if either $i$ and $j$ are connected by an undirected edge of $L_{c^{d}(\underline{\pi})}$ or if there is a directed edge of $L_{c^{d}(\underline{\pi})}$ pointing from $i$ to $j$.

Now $c^{d}(\underline{\pi})_{<}$is a partial permutation matrix, with nonzero entries in coordinates $(i, j)$ with $i<j$, and either $i$ and $j$ connected by an unmatched edge of $L_{c^{d}(\underline{\pi})}$ or a directed edge of $L_{c^{d}(\underline{\pi})}$ pointing from $i$ to $j$ (in this case the edge points from the smaller number to the larger number.) In particular, when we construct $\underline{\pi^{*}}, L_{\pi^{*}}$ has undirected edges corresponding to the undirected edges of $L_{c^{d}(\underline{\pi})}$ and the directed edges of $L_{c^{d}(\underline{\pi})}$ that point from a smaller number to a larger numbers. 
From the above discussion, the only reason why $c^{d}(\underline{\pi})$ might not be obtained from $\underline{\pi^{*}}$ by setting certain nonzero entries of $\pi^{*}$ to zero is that fixed points of $\pi^{*}$ aren't matched together by directed edges in the proper way. Let $a_{t_{1}}<\cdots<a_{t_{j}}$ be the fixed points of $\pi$ that when rotated by $c$ give rises to to the fixed points of $\pi^{*}$ (so $\left[\left[a_{t_{1}}+d\right]\right], \ldots,\left[\left[a_{t_{j}}+d\right]\right]$

are the fixed points of $\pi^{*}$.) In $L_{\underline{\pi}}$ there is a directed arrow from $a_{t_{m}}$ to $a_{t_{j-m+1}}$ for $\left\lfloor\frac{j}{2}\right\rfloor \leqslant m \leqslant j$; this implies that the there is a directed arrow from $\left[\left[a_{t_{m}}+d\right]\right]$ to $\left[\left[a_{t_{j-m+1}}+d\right]\right]$ for $\left\lfloor\frac{j}{2}\right\rfloor \leqslant m \leqslant j$. We must show $\left[\left[a_{t_{1}}+d\right]\right]<\cdots<\left[\left[a_{t_{j}}+d\right]\right]$ if it does, this means that the directed edges in $L_{\pi^{*}}$ agree with the directed edges of $L_{c^{d}(\underline{\pi})}$ on this set, and we are done. No matter what, we have $\circlearrowleft\left(\left[\left[a_{t_{1}}+d\right]\right], \ldots,\left[\left[a_{t_{j}}+d\right]\right]\right)$, since we took a cyclic ordered set and rotated it. To finish, we observe $\left[\left[a_{t_{1}}+d\right]\right]<\left[\left[a_{t_{j}}+d\right]\right]$, otherwise we would have replaced that directed edge with an undirected edge in creating $L_{\underline{\pi^{*}}}$, and thus we can conclude that $a_{t_{1}}+d<\cdots<a_{t_{j}}+d \bmod N$ as desired.

The final statement follows immediately from the second statement.

\section{Equations for the $\overline{F_{\pi}}$ 's}

The geometric description of the $\overline{F_{\pi}}$, s allows one to construct equations satisfied by these varieties. We have the following generalization of a theorem of Knutson and Zinn-Justin [1]. (Because the proofs of the theorems are identical, we refer the reader to their paper for both the proof and a description of the strip model mentioned below.)

Proposition 1 Fix an involution $\pi \in S_{N}$. The variety $\overline{F_{\pi}}$ satisfies the following equations:

(1) $M \bullet M=0$.

(2) (diagonal conditions) $\left(M^{2}\right)_{i i}=0$ if $i$ is a fixed point of $\pi$. (Notice that this equation is defined in terms of ordinary matrix multiplication, not in terms of $\bullet$.)

(3) (more diagonal conditions) $\left(M^{2}\right)_{i i}=\left(M^{2}\right)_{\pi(i) \pi(i)}$ if $i$ is not a fixed point of $\pi$

(4) for any $(i, j), r_{i j}(M) \leqslant r_{i j}(\underline{\pi})$. (equivalently, require the vanishing of all $r_{i j}(\underline{\pi})+1$ minors of the submatrix southwest of $(i, j)$ in the strip model of Knutson and Zinn-Justin.

We conjecture that these equations define the $F_{\pi}$ as a reduced scheme. As supporting evidence, we have the following:

Theorem 5 Let $\pi, \pi^{\prime}$ be two involutions in $S_{N}$, and suppose that $\pi$ has $k$ 2-cycles $\left(i_{1}, j_{1}\right), \ldots\left(i_{k}, j_{k}\right)$ and $N-2 k$ fixed points $1 \leqslant a_{1}<a_{2}<\cdots<a_{N-2 k} \leqslant N$. Then $F_{\pi} \subset \overline{F_{\pi^{\prime}}}$ if and only if

a. Every two cycle $\left(i_{l}, j_{l}\right)(1 \leqslant l \leqslant k)$ of $\pi$ occurs in the disjoint cycle decomposition of $\pi^{\prime}$.

b. Every two cycle occurring in the disjoint cycle decomposition of $\pi^{\prime}$ is either of the form $\left(i_{l}, j_{l}\right)$ or of the form $\left(a_{i}, a_{N-2 k+1-i}\right)$ for some $1 \leqslant i \leqslant\left\lfloor\frac{N-2 k}{2}\right\rfloor$. 
For the if direction, notice conditions (a) and (b) imply that $\underline{\pi} T \subset \underline{\pi^{\prime}} T$ and then the statement follows immediately from Theorem 3.

Conversely, suppose $F_{\pi} \subset \overline{F_{\pi^{\prime}}}$. Note that for all $t \in T,(\underline{\pi} t)_{i i}^{2}=t_{i} t_{\pi(i)}$ if $i \neq \pi(i)$ and $=0$ if $i$ is a fixed point of $\pi$. Now the equations from Theorem 4 hold on $\overline{F_{\pi^{\prime}}}$. Suppose that $i$ is a fixed point of $\pi^{\prime}$. Then $\left(M^{2}\right)_{i i}=0$ on $F_{\pi^{\prime}}$ and since $\underline{\pi} t \in \overline{F_{\pi^{\prime}}}$, we have $(\underline{\pi} t)_{i i}^{2}=0$ as well for all $t \in T$. By the previous computation $i$ must be a fixed point of $\pi$ also (otherwise $t_{i} t_{\pi(i)}$ is generically nonzero.) Suppose $(i, j)$ is a 2-cycle of $\pi^{\prime}$. Then $\left(M^{2}\right)_{i i}=\left(M^{2}\right)_{j j}$ on $\overline{F_{\pi^{\prime}}}$, and thus for all $\underline{\pi} t$. By the previous calculation, the equality only happen if either $(i, j)$ is a 2-cycle of $F_{\pi}$ or $i$ and $j$ are both fixed points of $\pi$ (otherwise we are trying to set $t_{i} t_{\pi(i)}=0$ or $t_{i} t_{\pi(i)}=t_{j} t_{\pi(j)}$ where $(i, \pi(i)),(j, \pi(j))$ are distinct orbits of $\pi$.) In particular, this implies condition a.

So we may assume $\pi^{\prime}$ satisfies condition a. Now we show inductively that for each $1 \leqslant l \leqslant\left\lfloor\frac{N-2 k}{2}\right\rfloor$ that either $\left(a_{l}, a_{N-2 k+1-l}\right)$ is a 2 cycle of $\pi^{\prime}$ or both $a_{l}$ and $a_{N-2 k+1-l}$ are fixed points of $\pi^{\prime}$. By induction we may assume the statement is true for $l=1,2, \ldots, j-1$. Now look at the equation of type 4 as defined in Theorem 4 corresponding to the coordinates $\left(a_{N-2 k+1-j}, a_{j}\right)$ in $\underline{\pi}^{\prime}$. This gives a maximum for the rank of the corresponding matrix for any point in $\overline{F_{\pi^{\prime}}}$. By induction, the submatrices of $\underline{\pi}^{\prime}$ and $\underline{\pi}$ lying southwest of $\left(a_{N-2 k+1-j}, a_{j}\right)$ are identical except possibly at coordinate $\left(a_{N-2 k+1-j}, a_{j}\right)$, and that the rank of this pair of matrices is equal if and only if $\underline{\pi}^{\prime}$ is nonzero in position $\left(a_{N-2 k+1-j}, a_{j}\right)$; if the rank is not equal that $\underline{\pi}$ has a bigger rank and cannot be contained in $\overline{F_{\pi^{\prime}}}$, which is a contradiction. But the requirement that $\underline{\pi}^{\prime}$ is nonzero in position $\left(a_{N-2 k+1-j}, a_{j}\right)$ is exactly the requirement that either $\left(a_{j}, a_{N-2 k+1-j}\right)$ is a 2-cycle of $\pi^{\prime}$ or both of $a_{j}, a_{N-2 k+1-j}$ are fixed points of $\pi^{\prime}$, as desired.

Note that one can summarize the above theorem by $\overline{F_{\pi}} \subset \overline{F_{\pi^{\prime}}}$ if and only if the support of $\underline{\pi}$ is properly contained in the support of $\underline{\pi}^{\prime}$; here the support of a matrix is the set of coordinates that have a nonzero entry.

One may worry that the shift action $c$ might induce more equations on $\overline{F_{\pi}}$. But one can modify the above proof to show that for any $d$, if $c^{d}\left(\overline{F_{\pi}}\right) \subset \overline{F_{\pi^{\prime}}}$, then $\overline{F_{\pi^{*}}} \subset \overline{F_{\pi^{\prime}}}$, where $\pi^{*}$ was defined in section 4 . (One uses the diagonal conditions to force the undirected edges to imply that $\pi^{\prime}$ inherits 2-cycles from $c^{d}(\underline{\pi})$ as above, and the rank conditions as above on the directed edges; that is, one shows that the support of $\pi^{\prime}$ contains the support of $c^{d}(\underline{\pi})$ by using a cyclic shift of the proof of the above theorem, and then notes that any $\underline{\pi^{\prime}}$ which contains the support of $c^{d}(\underline{\pi})$ also contains the support of $\underline{\pi}^{*}$.) Thus we need only check that $\overline{F_{\pi^{*}}}$ doesn't induce any new equations on $c^{d}\left(\overline{F_{\pi}}\right)$ that we haven't already described, which follows from the fact that the support $c^{d}(\underline{\pi})$ is contained in the support $\underline{\pi^{*}}$, as shown in section 4 .

Finally, since the irreducible components of $E$ correspond to the maximal $F_{\pi}$ under closure, the conjecture of Knutson and Zinn-Justin follows immediately as a corollary.

Corollary 2 The Brauer loop scheme $E$ is equidimensional, with irreducible components indexed bijectively by involutions with maximal number of 2-cycles. For any $F_{\pi}$, there is a unique involution $\pi^{\prime}$ with maximal number of 2-cycles such that $F_{\pi} \subset \overline{F_{\pi^{\prime}}}$. 
Proof. For any $F_{\pi}$ that does not have a maximal number of 2-cycles, Theorem 4 describes how to construct a $\pi^{\prime}$ with maximal number of 2-cycles such that $F_{\pi} \subset \overline{F_{\pi^{\prime}}}$. Moreover, this construction is unique (if $\pi$ has fixed points $a_{1}<a_{2}<\ldots<a_{N-2 k}$, then $\pi^{\prime}=\pi\left(a_{1} a_{N-2 k}\right) \ldots\left(a_{\left\lfloor\frac{N-2 k}{2}\right\rfloor} a_{N-2 k+1-\left\lfloor\frac{N-2 k}{2}\right\rfloor}\right)$

\section{References}

[1] A. Knutson and P. Zinn-Justin. A scheme related to the brauer loop model. Advances in Mathematics, 214:40-77, 2007.

[2] A. Melnikov. B-orbits in solutions to the equation $x^{2}=0$ in triangular matrices. $J$. of Algebra, 223:101-108, 2000. 